\title{
X-band Photoinjector for a Chirped-Pulsed FEL
}

E. C. Landahl, R. M. Alvis, A. L. Troha, W. E. White, H. A. Baldis, K. Li, J. P. Heritage, C. H. Ho, N. C. Luhmann, Jr., F. V. Hartemann

This paper was prepared for submittal to the

RF-98 Workshop

Pajaro Dunes, CA

October 5-9, 1998

December 15, 1998

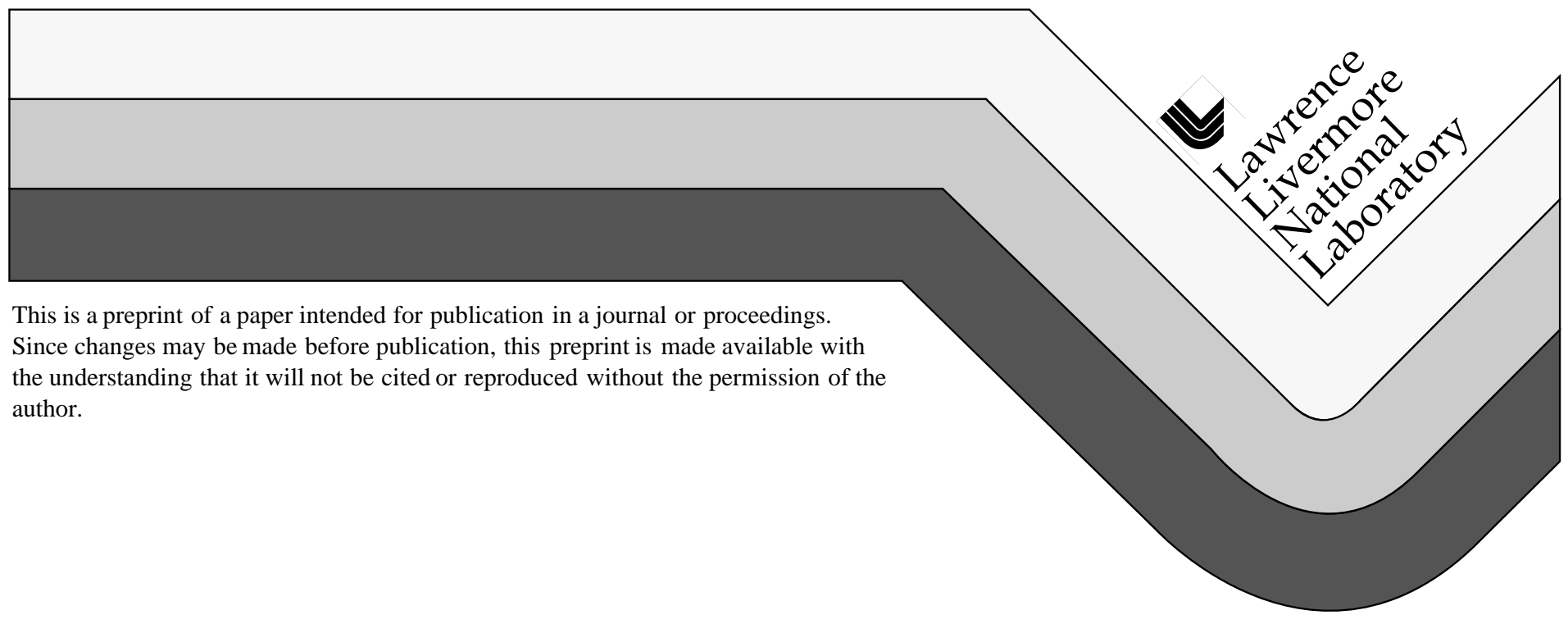


DISCLAMMER

This document was prepared as an account of work sponsored by an agency of the United States Government. Neither the United States Government nor the University of California nor any of their employees, makes any warranty, express or implied, or assumes any legal liability or responsibility for the accuracy, completeness, or usefuiness of any information, apparatus, product, or process disclosed, or represents that its use would not infringe privately owned rights. Reference herein to any specific commercial product, process, or service by trade name, trademark, manufacturer, or otherwise, does not necessarily constitute or imply its endorsement, recommendation, or favoring by the United States Government or the University of California. The views and opinions of authors expressed herein do not necessarily state or reflect those of the United States Government or the University of California, and shall not be used for advertising or product endorsement purposes. 
UCRL-JC-132730

\title{
X-band Photoinjector for a Chirped-Pulse FEL
}

\author{
E.C. Landahl ${ }^{1,2}$, R.M. Alvis ${ }^{1,2}$, A.L. Troha ${ }^{1,2}$, W. E. White ${ }^{3}$, \\ H.A. Baldis ${ }^{1,2}$, K. Li ${ }^{4}$, J.P. Heritage ${ }^{4}$, C.H. $\mathrm{Ho}^{5}$, N.C. Luhmann, Jr. ${ }^{1}$, and \\ F.V. Hartemann ${ }^{1.2}$ \\ 'Applied Science Department, University of California, Davis CA 95616 \\ ${ }^{2}$ Institute for Laser Science and Applications, LLNL, Livermore, CA 94550 \\ ${ }^{3}$ Lawrence Livermore National Laboratory, Livermore, CA 94550 \\ ${ }^{4}$ Electrical and Computer Engineering Department, University of California, Davis CA 95616 \\ ${ }^{5}$ Synchrotron Radiation Research Center, Taiwan
}

\begin{abstract}
The phase noise and jitter characteristics of the laser and rf systems of a high gradient X-band photoinjector have been measured experimentally. When $>100$ coherently phased $5 \mathrm{MeV}$ electron bunches are produced in bursts, the photoinjector should be an ideal electron source for a pulsed, pre-bunched free-electron laser (FEL) operating at $100 \mathrm{GHz}$. The laser oscillator is a self-modelocked Titanium:Sapphire system operating at the 108th subharmonic of the rf gun. The $\mathrm{X}$-band signal is produced from the laser by a phase-locked dielectric resonance oscillator, and amplified by a pulsed TWT and klystron. A comparison between the klystron and TWT amplifier phase noise and the fields excited in the if gun demonstrates the filtering effect of the high $Q$ structure, thus indicating that the rf gun can be used as a master oscillator, and could be energized by either a rf oscillator such as a magnetron or a compact source such as a cross-field amplifier. In particular, the if gun can play the role of a pulsed if clock to synchronize the photocathode laser system: direct drive of a synchronously modelocked AlGaAs quantum well laser has been achieved using the X-band gun rf fields. This novel, $\mathrm{GHz}$ repetition rate, sub-picosecond laser system is being developed to replace the more conventional femtosecond $\mathrm{Ti}: \mathrm{Al}_{2} \mathrm{O}_{3}$ system. Some advantages include pumping this laser with a stabilized current source instead of a costly, low efficiency pump laser. Finally, dark current measurements and initial photoelectron measurements are reported.
\end{abstract}

\subsection{INTRODUCTION}

Low laser and rf phase noise and jitter are essential to the operation of advanced photoinjectors [1]-[3] which have numerous applications ranging from laser acceleration [4], and biomedical applications, such as Compton scattering $\mathrm{X}$-ray sources [5]-[7], to chirped pulse free-electron lasers (FELs) [1], [8]-[10] for ultrawideband radars [11]. The theory of chirped pulse FELs and the production of coheent synchrotron radiaiton has been extensively studied [8]-[10]; therefore, the 


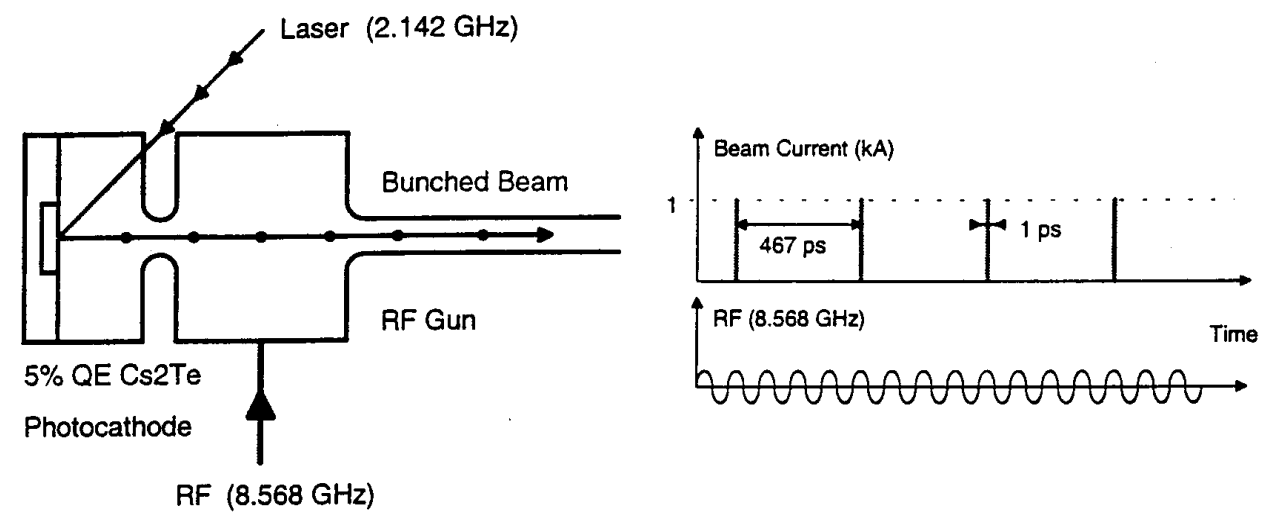

FIGURE 1. X-band photoinjector: basic principle.

focus of this paper will be the design and experimental operation of a high brightness $\mathrm{X}$-band photoinjector capable of producing relativistic prebunched electron beams that are ideally suited for chirped pulse FEL applications. In a photoinjector, photoelectrons are produced by a laser and accelerated in a high $\mathrm{Q}$ if structure supporting a TM mode, as shown schematically in Fig. 1. Furthermore, the use of a GHz repetition rate photocathode laser system will enable us to produce relativistic photoelectron bunches at the fourth subharmonic of the operating frequency of the rf gun. The synchronization between the laser and rf components is clearly of paramount importance for the optimization of the overall system performance. In the X-band photoinjector discussed here, one degree of rf phase corresponds roughly to $325 \mathrm{fs}$. Figure 2 shows simulations which predict degradation of the output electron beam characteristics with a variation of more than five degrees from the optimum injection phase. The simulations were based on a bunch charge of $0.1 \mathrm{nC}$, a temporal width of $0.9 \mathrm{ps}$ FWHM and a focal spot size of $0.67 \mathrm{~mm}$ HWHM. Here, both temporal and spatial profiles are assumed to be Gaussian. At an optimized injection phase of $65^{\circ}$, achieving these parameters at the photocathode should produce an electron beam with a normalized transverse rms emittance of $0.7 \pi \mathrm{mm}$-mrad with an energy of $5.7 \mathrm{MeV}$ for $16 \mathrm{MW}$ of RF power dissipated in the gun, and an axial energy spread of $0.25 \%$. At a charge of $1 \mathrm{nC}$ the emittance should increase only to $2.5 \pi \mathrm{mm}$-mrad.

Synchronization is also a key factor in producing trains of 100 or more coherently phased relativistic electron bunches in a single macropulse by using a $\mathrm{GHz}$ repetition rate modelocked $\mathrm{AlGaAs}$ semiconductor laser oscillator. By comparison with a conventional photoinjector, the luminosity of the output beam is then increased by at least two orders of magnitude. If the bunches are coherently phased within a few tens of femtoseconds, radiated powers can be generated in a freeelectron laser (FEL) which are orders of magnitude higher than those of single bunch devices. In addition, the means of synchronizing a second tunable laser by using a photoinjector driven FEL is important for use in pump-probe experiments as well as 


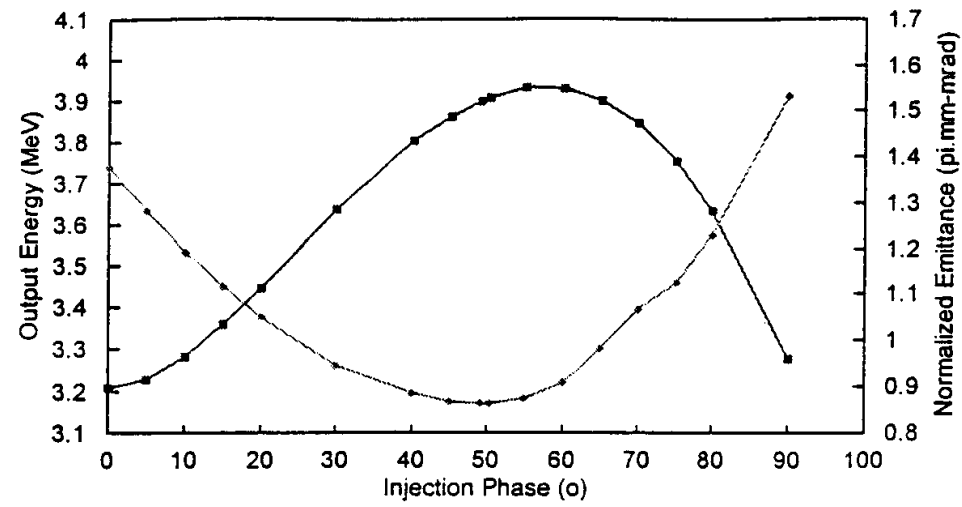

FIGURE 2. Normalized transverse emittance and rf gun output energy as functions of the laser injection phase angle. The other parameters are presented in the text.

a means for selecting single picosecond pulses from the FEL [12]. This capability of the photoinjector makes it a unique tool for nonlinear electron-photon interaction experiments, including ponderomotive [13] [14], Kapitza-Dirac [15] [16] and nonlinear Compton scattering [5]-[7], laser/FEL pump-probe experiments (femtosecond synchronization), and surface science (picosecond pump-probe nonlinear mixing) [17].

In this paper, we first present experimental measurements of the phase noise of different components, including a Titanium:Sapphire laser oscillator, an X-band traveling-wave tube amplifier (TWT), and a high power X-band klystron. We then demonstrate the filtering effect of the high $Q$ rf gun. This new result is very important, as it indicates that highly efficient, but relatively noisy if sources, such as a magnetron or a cross-field amplifier (CFA), could be used to drive a high gradient photoinjector. In addition, the highly phase stable if fields excited in the if gun can be used as a master oscillator for the entire system: in this way, the laser pulses can now be directly synchronized to the accelerating fields in the photoinjector.

This paper is organized as follows. In Sec. 2, the X-band gun design and cold tests performed on a prototype cavity are briefly discussed. Section 3 presents the if system used to provide high power to the gun. Characterization of a "dark current" electron beam, created when the gun is energized, is also reported. In Sec. 4 we discuss the two laser oscillator systems and the laser amplifiers used to produce photoelectrons. In Sec. 5 we present phase noise measurements of different components in the gun system and demonstrate phase noise reduction by the high-Q cavity. Finally, Sec. 6 describes the measurement of the first accelerated photoelectrons observed in the system. Conclusions are drawn in Sec. 7. 


\section{X-BAND GUN DESIGN AND COLD TESTS}

The photoelectron bunches are optimally accelerated by the $11 / 2$ cell structure when the peak fields in the two cells are nearly equal, as demonstrated by PARMELA simulations. Providing such a balanced field is accomplished through small changes in the outer radii of the full and half cells. The unbalanced case represents equal radii in the half and full cells with a resonant frequency of $8.548 \mathrm{GHz}$ in the whole structure. Slight variations in the half and full cell radii allow to balance the peak fields in the two cells, while maintaining the structure resonant frequency. In practice, the temperature of the accelerating structure will be used to adjust the overall resonance of the structure, while the balance of the fields can be accomplished through the tuning of either cell, or both.

After the geometry of the If accelerator cavity had been optimized through computer simulation, a cold test model was constructed from oxygen-free, high conductivity (OFHC) copper to test cavity resonance, coupling, $\mathrm{Q}$, and axial electric field profile, as well as the effects of the field tuners and monitors. The cold test cavity parts were machined at the Synchrotron Radiation Research Center (SSRC) facility using computer controlled, high precision milling machines to a tolerance of 0.01 $\mathrm{mm}$, with inner cavity surfaces polished to $1.6 \mu \mathrm{m}$.

The quality factor of the cold test cavity was determined using the impedance method, adapted to the use of a network analyzer. Basically, the impedance of the accelerator cavity as a load is measured as a function of frequency near resonance.

$A Q_{0}$ value of 4,471 was measured. This value was confirmed by measurements taken on a separate occasion at SRRC. There the $-3 \mathrm{~dB}$ points were used to calculate a $Q_{0}$ value of 4,470 at a resonant frequency of $8.4935 \mathrm{GHz}$. The ohmic or unloaded cavity $\mathrm{Q}\left(\mathrm{Q}_{0}\right)$ value calculated by SUPERFISH was 7,168 . Since the computer code only takes into account the cavity itself and not the coupled waveguide feed, this $Q$ value is expected to be about twice the measured value at critical coupling. The $Q$ of the high power, brazed cavity structure falls well above the $Q$ measured for the cold test cavity, and is bounded on the high side by the SUPERFISH model, which includes no coupling and no imperfections in material or construction.

\section{HIGH POWER TESTS AND DARK CURRENT MEASUREMENTS}

The current gun design requires $11 \mathrm{MW}$ of X-band rf power to achieve a 150 $\mathrm{MV} / \mathrm{m}$ average accelerating gradient. The corresponding peak cathode field is 350 $\mathrm{MV} / \mathrm{m}$ which is below the expected breakdown limit of $570 \mathrm{MV} / \mathrm{m}$. The dielectric resonance oscillator (DRO) converts the output of a frequency synthesizer from $79.138 \mathrm{MHz}$ to the 108th harmonic, $8.546904 \mathrm{GHz}$. The TWTA is air cooled and 
uses periodic permanent magnet focusing to provide a $6 \mu$ s pulse of $\mathrm{rf}$ at a $1 \mathrm{~kW}$ level and a $30 \mathrm{~Hz}$ repetition rate. This signal is fed to the klystron through standard WR-112 waveguide. The klystron was originally designed for SLAC development efforts and has a measured peak response at $8.845 \mathrm{GHz}$. It is energized by a $\mathrm{HV}(350$ $\mathrm{kV}, 2 \mu \mathrm{s}$ ) pulse forming network (PFN) type modulator, using a hydrogen thyratron switch.

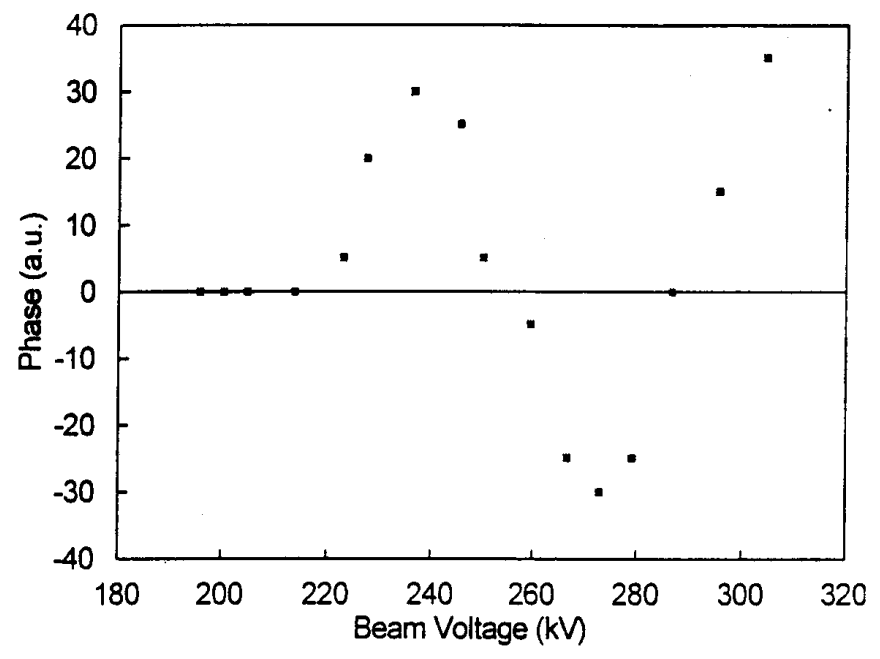

FIGURE 3. Klystron phase variation with voltage.

This rf system has advantages for optimizing the photoinjector. For trains of 100 photoelectron bunches the effects of beam-loading, wakefields, and bunch-tobunch interactions can become significant. The capability of providing up to $20 \mathrm{MW}$ from the klystron means that the cavity $Q$ can be decreased by overcoupling, thereby reducing beam loading effects. Also, the klystron power can be modulated by shaping the rf drive pulse from the TWTA, allowing adjustments to keep the gun output energy constant over the bunch train.

The klystron was characterized with its gun energized at $300 \mathrm{kV}$ and $135 \mathrm{~A}$. The power output peaks at approximately $11 \mathrm{MW}$. The $-3 \mathrm{~dB}$ bandwidth is $50 \mathrm{MHz}$. Figure 3 shows the klystron phase variation at $5 \% \mathrm{kV}$. This variation is inherent in the klystron design. The plasma wavelength of the electron beam in the klystron increases with higher voltage. At nominal operating voltage, the length of the klystron interaction circuit is approximately equal to one plasma wavelength. When the applied voltage changes, the klystron dimensions no longer match the plasma wavelength and a phase shift is introduced. Modeling of this X-band klystron correctly predicts the observed phase shift value. Voltage-induced phase variations in the klystron gives another reason to synchronize the laser directly to the fields inside the rf gun: any adjustments to the rf system affecting the klystron power output will 
modify the phase, thus requiring immediate response of the laser system during photoinjector optimization procedures.

Coupling and $\mathrm{Q}$ measurements were done by measuring reflection of if from the gun. Maximum coupling is achieved near the peak klystron power frequency. The $-3 \mathrm{~dB}$ bandwidth is now approximately $2 \mathrm{MHz}$, corresponding to a $\mathrm{Q}$ of 4,270. The coupling into the gun's half-cell is measured at 3,560 , which differs only slightly from the SUPERFISH simulation value of 3,584.

A simple pillbox model of the cavity predicts a linear shift in the $\mathrm{TM}_{010}$ resonant frequency as the gun dimensions change with temperature. The $\mathrm{TM}_{010}$ resonant frequency is

$$
f_{0}=\frac{c}{2 \pi} \frac{\chi_{01}}{a}
$$

where $c$ is the speed of light, $\chi_{01}$ is the first root of the zero order Bessel function, and $a$ is the cavity radius. Using the linear coefficient of thermal expansion for Copper, $\alpha=1.610^{-5}{ }^{\circ} \mathrm{C}^{-1}$, the slope of the frequency shift can be calculated to be -136 $\mathrm{kHz} /{ }^{\circ} \mathrm{C}$ as follows:

$$
\Delta f=\frac{d f}{d a} \frac{d a}{d T} \Delta T \cong-f_{0} \alpha \Delta T
$$

A temperature change is seen experimentally as more power is coupled into the gun. Figure 4 measures this frequency change slope to be $-160 \mathrm{kHz} /{ }^{\circ} \mathrm{C}$; in good agreement with the calculated value.

The negative slope of frequency vs. temperature unfortunately means that the resonance frequency of the cavity, which when cold is already lower than the peak power output frequency of the klystron, will only move farther away from peak

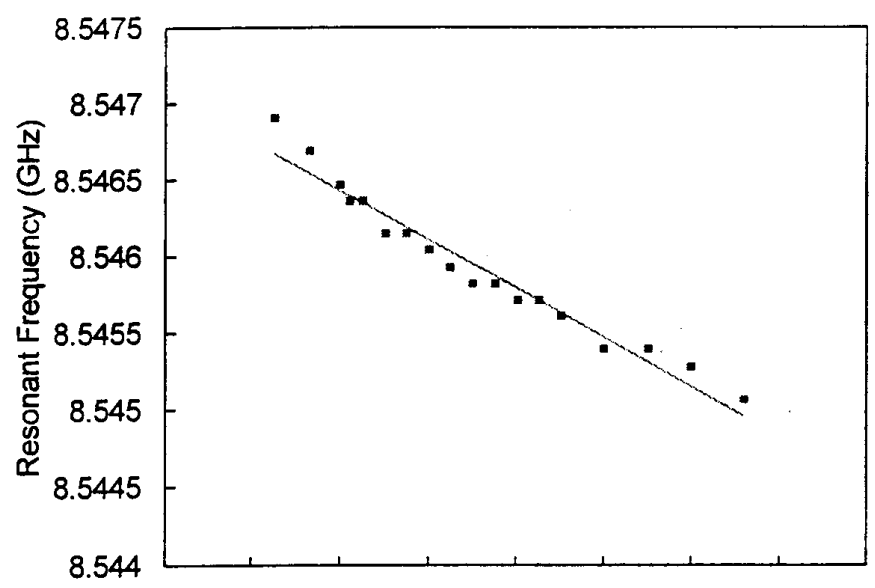

FIGURE 4. Measured X-band rf gun resonant frequency as a function of temperature. 
power coupling as the gun heats up. Eventually an equilibrium point is reached where the klystron power is low enough that the gun temperature no longer increases. Operating at low ( 1 to $3 \mathrm{MW}$ ) power also has to be done with this rf system to protect the klystron from reflection damage. This could occur when the cavity shorts during a plasma breakdown, reflecting all incident power back to the klystron. The 1-2 $\mu$ s long flat-top klystron pulse is long enough for the reflected and incident waves to interfere in a standing wave pattern at the klystron window input, generating fields that are strong enough to potentially damage the window. The low powers used here means breakdown is less likely to occur, and when it does, the power levels reflected back to the klystron window are not dangerously high. High power operation will be possible with the next rf gun prototype. The cold cavity resonance will be at the maximum klystron power output, and the cavity will be kept at this frequency by forced cooling through the cavity exterior sufficient to carry away $300 \mathrm{~W}$ of heat. In addition the danger of reflection will be mitigated by installation of a unique $\mathrm{X}$-band magic tee in the waveguide which will provide $6 \mathrm{~dB}$ of isolation between the cavity and klystron.

The prototype photoinjector cavity was processed at a $30 \mathrm{~Hz}$ rf pulse repetition rate. The attenuation from the TWTA was stepped from $-20 \mathrm{~dB}$ to $-10 \mathrm{~dB}$ while the vacuum was monitored. In case of breakdown, the vacuum increases from $10^{-9}$ torr to $10^{-7}$ torr as plasma is created inside the gun. A Faraday cup at the end of the gun simultaneously shows a large collected current when this happens. Initial processing was complete when an attenuation of $-10 \mathrm{~dB}$ ( $2 \mathrm{MW}$ coupled into the rf gun) was achieved at continuous low pressure.

Plasma discharge is not the only source of electrons in the energized gun. Electrons can also be pulled off of the cathode by strong if fields without the presence of a laser. This dark current is characterized in the X-band gun using a fluorescent screen placed in the beamline. As opposed to the sporadic bright flashes seen during plasma discharge in rf breakdown, dark current is seen steadily when the power coupled into the cavity exceeds $1 \mathrm{MW}$. Unfocused, the dark current appears as a constant "smear" across the screen. When a $1 \mathrm{~mm}$ aperture is placed in the beam, a dot can be produced which is focused using solenoid magnets and steered using dipole magnets. From the Fowler-Nordheim equation,

$$
I \propto \frac{E_{0}^{2} \cos ^{2} \phi}{W} \exp \left[-\frac{B W^{3 / 2}}{E_{0} \cos \phi}\right]
$$

(where $B$ is a constant and $W$ is the work function of the cathode) it is clear that the dark current, $I$, is strongly peaked when the electric field, $E_{0} \cos \phi$, is a maximum on the cathode at the back of the cavity. At this $0^{\circ}$ phase angle the fields in the cavity are not timed for optimal acceleration, so the electron energy measured should be much lower than what is achievable with photoelectrons injected at the proper phase. This electron energy was estimated using dipole steering with an apertured beam. The dipole strengthcan be used to calculate the energy of the electrons by measuring 

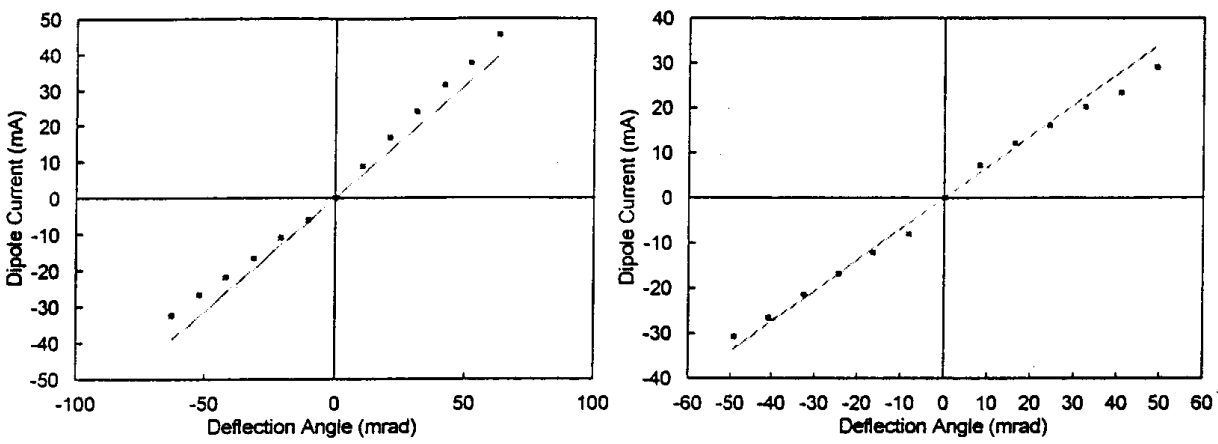

FIGURE 5. Dipole scans of the dark current beam. Horizontal deflection (left) gave an energy of $321 \mathrm{keV}$ for the electrons; vertical deflection (right) gave an energy of $276 \mathrm{keV}$.

the deflection angle (Fig. 5). Dipole scans using horizontal and vertical steering magnets gave energies of $321 \mathrm{keV}$ and $276 \mathrm{keV}$, respectively. During this measurement the apertured beam profile stayed constant over different dipole strengths, indicating that the electrons had a fairly narrow energy spread. This fact further supports the conclusion that these electrons are Fowler-Nordheim dark current, emitted in a narrow time window when the rf phase is close to $0^{\circ}$, and also indicates that greater energies will be obtained for photoelectrons emitted at the optimum phase angle for acceleration. Figure 6 shows an example where the dark current measurements are modeled using PARMELA. Electrons were injected at $0^{\circ}$ in the rf cycle and propagated through the cavity at different power levels. The energies have a local maximum of nearly $350 \mathrm{keV}$ a little above $1 \mathrm{MW}$, in agreement with experiment. The simulation also explains the sudden onset of dark current at $1 \mathrm{MW}$, since below this power level the electrons do not make it to the full cell quickly enough and experience deceleration back towards the cathode after the fields have reversed. Attempts

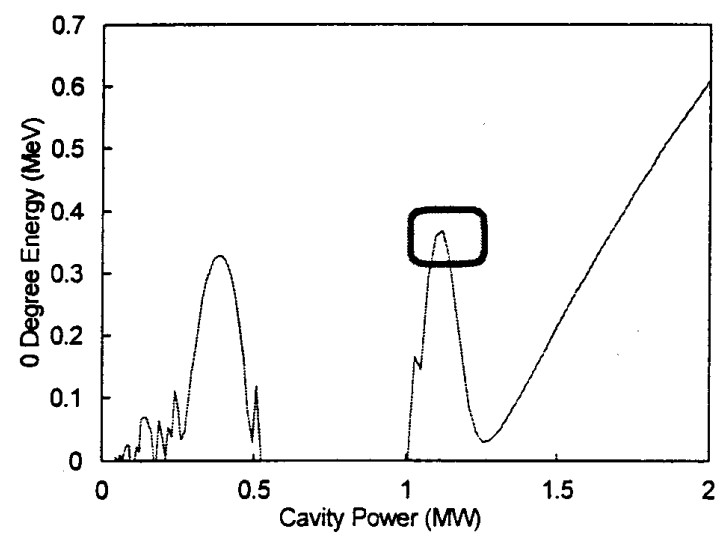

FIGURE 6. PARMELA simulation of gun output electron energies for electrons that were initially placed at the cathode with $0^{\circ}$ phase $\mathrm{RF}$, for different values of input power. Outlined region shows where dark current was measured. 
to measure the absolute charge in the dark current using a Faraday cup were made difficult because of rf noise levels, and because the integrated charge is relatively small (typically in the $\mathrm{pC}$ range).

The 1.5 cell $\mathrm{X}$-band $5 \mathrm{MeV}$ photoinjector requires $11 \mathrm{MW}$ of rf power for optimum operation. A planned upgrade to a combined $20 \mathrm{MeV}$ plane wave transformer linac / photocathode (done in collaboration with UCLA and DULY Research, Inc.) will require in excess of $20 \mathrm{MW}$ of rf power. Medium power results (below saturation) are shown in Figure 7.
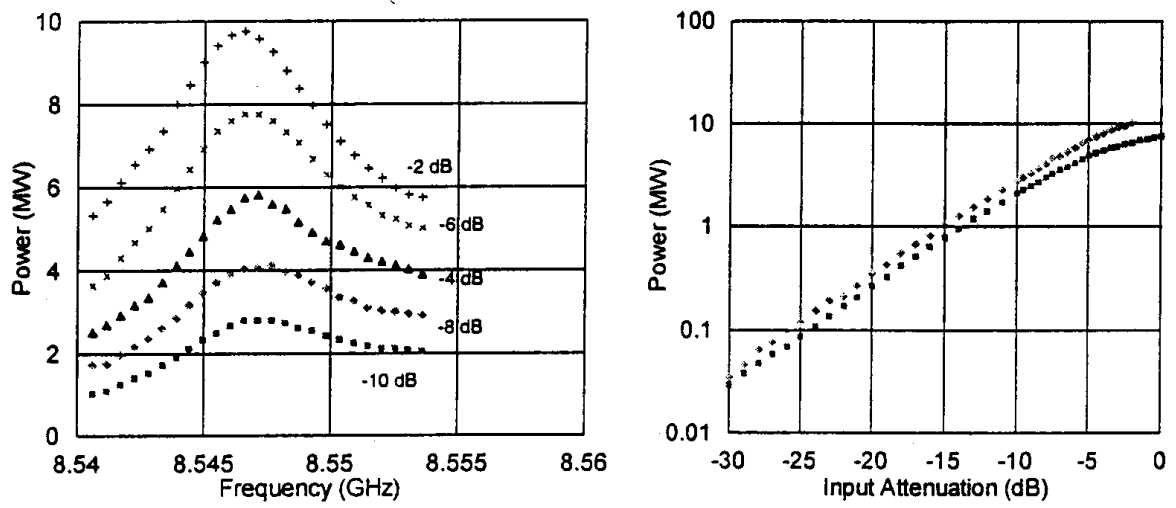

FIGURE 7. Power measurements on the klystron at $20 \mathrm{~Hz}$ were made using a calorimeter head placed on a calibrated directional coupler (total attenuation of $-49.25 \mathrm{~dB}$ ) placed before a water cooled matched load. Left: Klystron tuning at nominal beam voltage $(330 \mathrm{kV})$ and current (155 A). TWTA input is adjusted from $-10 \mathrm{~dB}$ to $-2 \mathrm{~dB}$ as indicated. Right: Klystron transfer curve at subnominal (lower trace: $300 \mathrm{kV}, 135 \mathrm{~A}$ ) and nominal (upper trace). Note that saturation is not reached, even at below nominal beam voltages.

\section{LASER SYSTEMS}

To get the most advantages of the X-band photoinjector system, the cathode should be illuminated with the shortest laser light pulses possible at high UV energies in order to generate short, low energy spread, high current electron bunches. In addition to the desired high repetition rate of the laser on the cathode during the if pulse (up to one laser pulse every few rf cycles), the timing jitter between these pulses should be less than one degree of rf phase. To achieve these goals, two different laser systems have been used. While a unique multiple quantum well (MQW) saturable absorber modelocked AlGaAs laser oscillator system is being developed which has the potential of meeting all of the above criteria, a commercial $\mathrm{Ti}: \mathrm{Al}_{2} \mathrm{O}_{3}$ modelocked laser system is also being used to provide single laser shots to generate photoelectrons.

The integration of a compact semiconductor laser oscillator in the rf photoinjector system is particularly attractive for the following two reasons. First, this oscillator operates in the $800-900 \mathrm{~nm}$ wavelength range and can therefore be readily used to drive the $\mathrm{Ti}: \mathrm{Al}_{2} \mathrm{O}_{3}$ multipass amplifier which produces the required laser pulse 


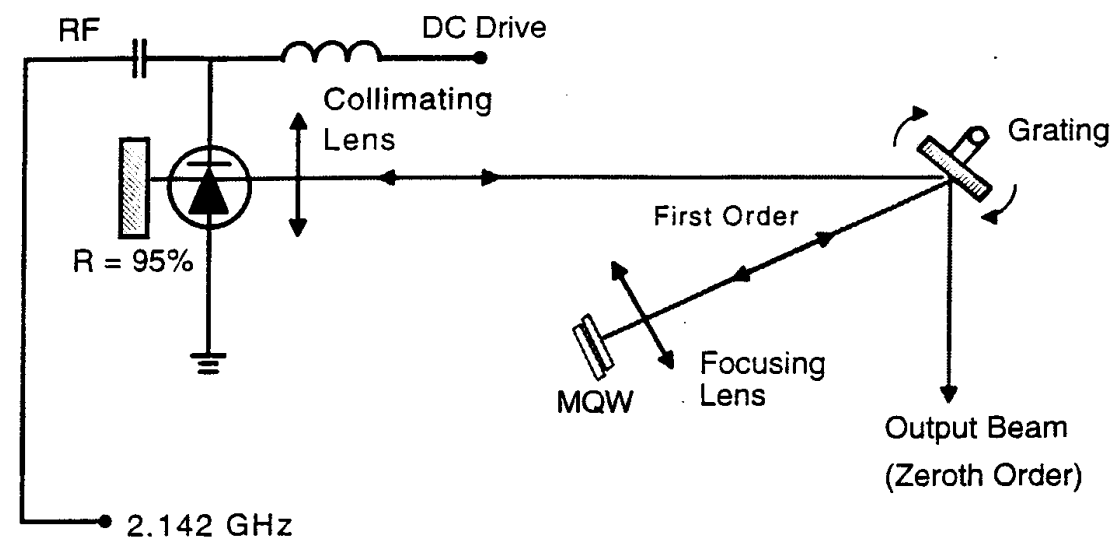

FIGURE 8. Schematic of the AIGaAs synchronously modelocked oscillator.

energy for the photocathode. The other outstanding feature of this laser is the fact that it is modelocked to an external clock with an extremely low jitter and can therefore be synchronized to the rf gun with unprecedented accuracy. Relative timing jitter of $\sim 400$ fs rms over a measurement interval of $100 \mathrm{~s}$ has been inferred from direct measurements of the linewidth of the forty-first harmonic of the if power spectrum of the periodic pulse train [18] from a 450 ps pulsewidth version of his laser. This result is typical of other modelocked semiconductor lasers [19], [20] and will ensure the production of periodic electron bunches that are highly coherent at the if gun frequency. This number translates to a $\sim 10^{-2}$ fs calculated jitter over the 50 -ns UV pulse train (well below any measurable value!). The phase stability of an actively modelocked semiconductor laser is vastly superior in large part because of the stability of direct electrical pumping in a pn junction. Low-noise high-stability electrical signals (both dc and rf) are used along with saturable absorbers to modelock semiconductor lasers. The direct connection between electrical injection and optical gain in a semiconductor laser results in very low timing jitter during externally modelocked operation. In addition to the very low jitter, this laser offers at least four additional benefits: 1) high energy efficiency, 2) compactness, 3) potentially enhanced ease of operation on a day-to-day basis, and 4) very low operating costs. The oscillator is shown in Fig. 8. An AlGaAs/GaAs semiconductor diode amplifier provides gain for an external optical resonator. The diode is antireflection (AR) coated to suppress oscillations from facet reflections. The diode is dc biased just below threshold and a high spectral purity of drive is superimposed with a bias insertion tee. The resulting precise gain switching provides jitter free production of synchronized optical pulses. A stagger tuned stack of multiple quantum well (MQW) material is AR coated, lifted off its substrate, and affixed to the high-reflection mirror at the other end of the resonator. The MQW structure consists of alternating layers of AlGaAs (10 nm thick, doped with 30\% Al) and GaAs (7 nm thick). The MQW used in the laser system described here has 100 layer pairs. The stagger tuning yields a broadband absorber 
necessary to support pulses as short as 100 to $200 \mathrm{fs}$. A dose of $200 \mathrm{keV}$ protons serves to partially damage the $\mathrm{QW}$ material, thereby decreasing the carrier lifetime so that the absorption recovery is very rapid to ensure good pulse shortening by the absorber. Lenses couple the beam into the guiding region in the diode and onto the absorber. The four-prism arrangement serves to control the spectrum and introduce moderate group velocity dispersion compensation.
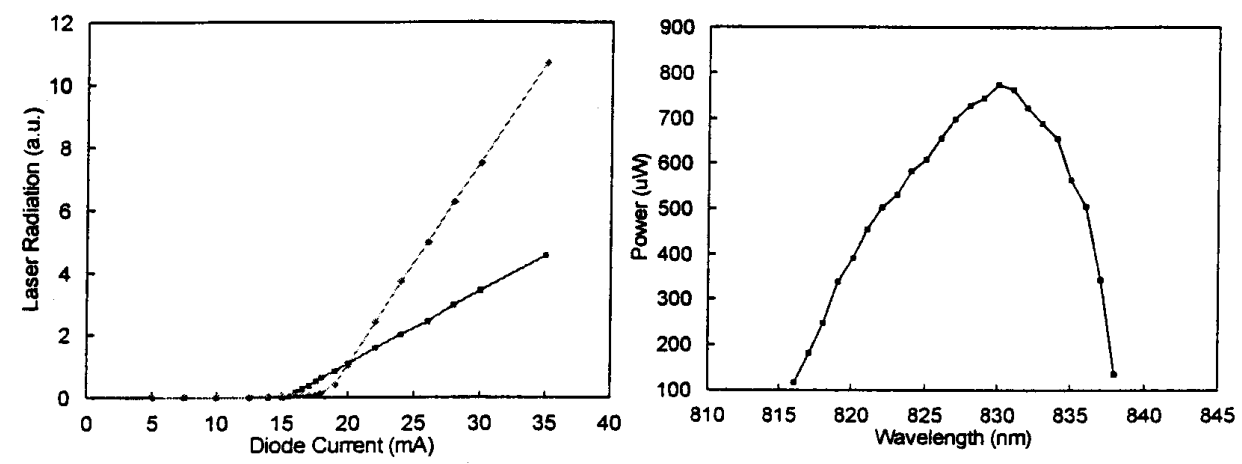

FIGURE 9. Left : Light versus current diode characteristic, with and without an external cavity. Right : tuning curve of the laser diode.

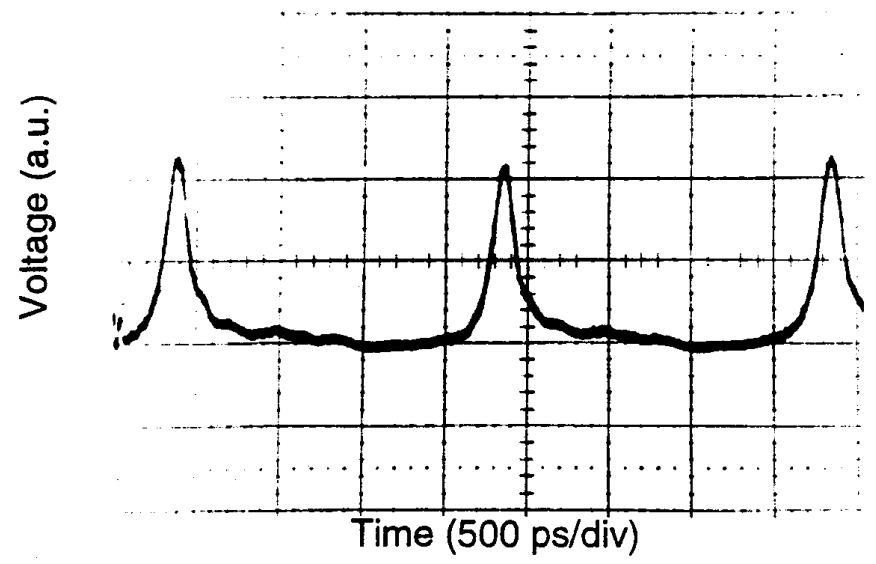

FIGURE 10. Modelocked pulse train from the laser diode modulated at $1.462 \mathrm{GHz}$.

Figure 9 (left) shows the light-current characteristic of the AlGaAs laser diode used in these experiments, both with and without an external cavity. Lasing in the external cavity is demonstrated by the lower threshold current observed. The optical bandwidth of the diode is also shown in Fig 9 (right). Lasing is observed between 820 and $835 \mathrm{~nm}$, with a maximum at $830 \mathrm{~nm}$. This curve is obtained by replacing the external cavity mirror by a grating, thus allowing for selective wavelength feedback; it indicates that the optical bandwidth is sufficient to support pulses as short as 
150 fs. Depending on If power levels, either high frequency modulation or modelocking (Fig. 10) is obtained from the laser. Initial modelocking results have demonstrated $<50$ ps HWHM pulses at a repletion rate of $0.487 \mathrm{GHz}, 0.981 \mathrm{GHz}$, and $1.462 \mathrm{GHz}$, depending on the cavity length and dc pump current. Note that on the oscilloscope traceS shown in Fig. 10, the pulse duration is dominated by the photodiode response, approximately $50 \mathrm{ps}$. Shorter pulses, obtained with the MQW, will be amplified by the eight-pass laser, described in the next paragraphs. Research into the dynamics of such lasers have shown that they can not generate a 250 -fs pulse directly because of an ultrafast gain depletion mechanism that arises for pulses below 1 ps in duration. However, very broadband but long $(\sim 5 \mathrm{ps})$ linearly chirped pulses can be produced which are easily compressed. It may be possible to produce an optimized stretched pulse directly from the resonator, eliminating need for a separate set of grating pulse stretchers; this will greatly reduce system size when combined with the Ti: $\mathrm{Al}_{2} \mathrm{O}_{3}$ amplifier.

To achieve the high gain needed to amplify the output of the diode oscillator $(10-100 \mathrm{pJ})$ to the $100 \mathrm{~mJ}$ level, there are two options. These gains can be achieved either by a regenerative amplifier or by a multipass amplifier. To use a regenerative amplifier for a $100 \mathrm{~ns}$ burst, one requires a stable optical cavity that is at least as long as the pulsewidth: $30 \mathrm{~m}$. This cavity length is prohibitive, so we opted for a multipass amplifier design. In addition, the laser amplifier must have sufficient optical bandwidth to support 250 fs duration pulses and yield high beam quality. Using these two criteria, the best choice is a frequency-doubled Nd:YAG-pumped chirped pulse Ti: $\mathrm{Al}_{2} \mathrm{O}_{3}$ amplifier. For this type of amplifying medium, single-pass gains of eight are easily achieved at a wavelength of $830 \mathrm{~nm}$. The amplifier is configured such that the pulse train passes through the gain medium eight times. This is achieved with a three-dimensional (3-D) "bow-tie" configuration, first demonstrated at Lawrence Berkeley Laboratory (LBL) using $161 \mathrm{~m}$ radius of curvature mirrors in a confocal configuration in which the $\mathrm{Ti}: \mathrm{Al}_{2} \mathrm{O}_{3}$ rod is placed at the focal point of the mirrors. In this manner, many passes are achieved through geometry rather than switching the pulse train into a long stable optical cavity. With a small-signal singlepass gain of eight, this corresponds to an overall gain of $-72 \mathrm{~dB}$. For an input energy of $10 \mathrm{pJ}$ per pulse, this amplifier will yield an output of $168 \mathrm{~mJ}$ per pulse, as long as gain depletion and upper state lifetime effects are ignored. These effects can be overcome if one tailors the amplitude of the input pulse train such that the pulses at the beginning of the train are of lower intensity than the later ones. This compensation is achieved using a commercially available integrated optical modulator. The input pulse train is coupled into an integrated $\mathrm{LiNbO}_{3}$ Mach-Zender device via single mode fiber pigtails, and the intensity is modulated by a $5 \mathrm{~V}$ signal with a bandwidth of 5 GHz. In this manner, two important goals are achieved simultaneously. The signal is modulated with a fast low-voltage feed-forward loop, and is also spatially filtered by the single mode fiber. In addition, the UV quadrupler output pulses can be sampled and compared to a reference signal to activate an additional fast low-voltage feedback loop guaranteeing an extremely stable output pulse train. 
For initial photoinjector experiments, a commercial modelocked laser system is used to produce $80 \mathrm{MHz}$ trains of pulses from which single pulses are selected at $10 \mathrm{~Hz}$ to go through a regenerative amplifier system. A Spectra Physics Millennia laser head was used to pump a Tsunami laser oscillator. Although this system is passively modelocked, a lock-to-clock option allows the short pulses (measured $<80$ fs at a center wavelength of $825 \mathrm{~nm}$ ) to be locked to an external oscillator with a measured timing jitter of $<1$ ps rms. After stretching with a grating pair, the selected pulse was sent through a regenerative amplifier using the same $\mathrm{Ti}: \mathrm{Al}_{2} \mathrm{O}_{3}$ crystal pumped by the same frequency double Nd:YAG laser as used in the eight pass system previously discussed. The regenerative amplifier does not have to be very large for a single shot and it was easier to work with for these first experiments. Again, eight passes of the single shot were allowed before the pulse was switched out of the cavity to the grating compressor and nonlinear crystals. Energy measurements after compression showed over $200 \mu \mathrm{J}$ was contained in each pulse. At an expected UV conversion efficiency of $1 \%$, about $0.03 \mathrm{nC}$ of photoelectrons should be available for acceleration. Improvement of laser amplification systems and frequency conversion efficiency should make possible creation of far greater amounts of charge.

\section{PHASE NOISE AND JITTER SUPPRESSION EXPERIMENTS}

Phase noise from the rf system comes from a number of sources. A major contributor is the variation in modulator voltage during the gun bias pulses. Amplification in both the TWTA and the klystron results from the modulation of the electron beam, so any additional modulation of the beam velocity due to the bias pulses directly alters the rf output. The interaction circuit of the TWTA is a transmission line rather than a resonant structure, so this device has a particularly large bandwidth. Although useful for tuning over large frequencies and for creating sharp-edged pulses, the large bandwidth also means a large amount of noise can also be amplified. Measurements showed the TWTA to have a bandwidth corresponding to a potential phase slew of $57.3 \%$ ms. Actual phase noise should be much less than this because the TWTA can be adjusted and optimized for a quiet signal. The klystron is a resonant structure but only has a $Q$ of 285 , corresponding to a phase slew of $1.72 \%$ ns. As a high power amplifier, it is likely to be a major source of phase noise in the rf system. In particular, noise in the high voltage source will cause the characteristics of the electron beam in the klystron to change. Since this beam is the amplifying element, any changes in its properties can be expected to have a strong effect in the output rf. The only structure in the system with high enough $Q$ to achieve considerable noise filtering is the photoinjector cavity itself. The loaded $\mathrm{Q}$ of the high power cavity is 3,584 resulting in a phase slew rate of $0.14^{\circ} / \mathrm{ns}$. Larger phase variations over a shorter time scale will be essentially reflected by the cavity load, and will not significantly affect the cavity fields. 

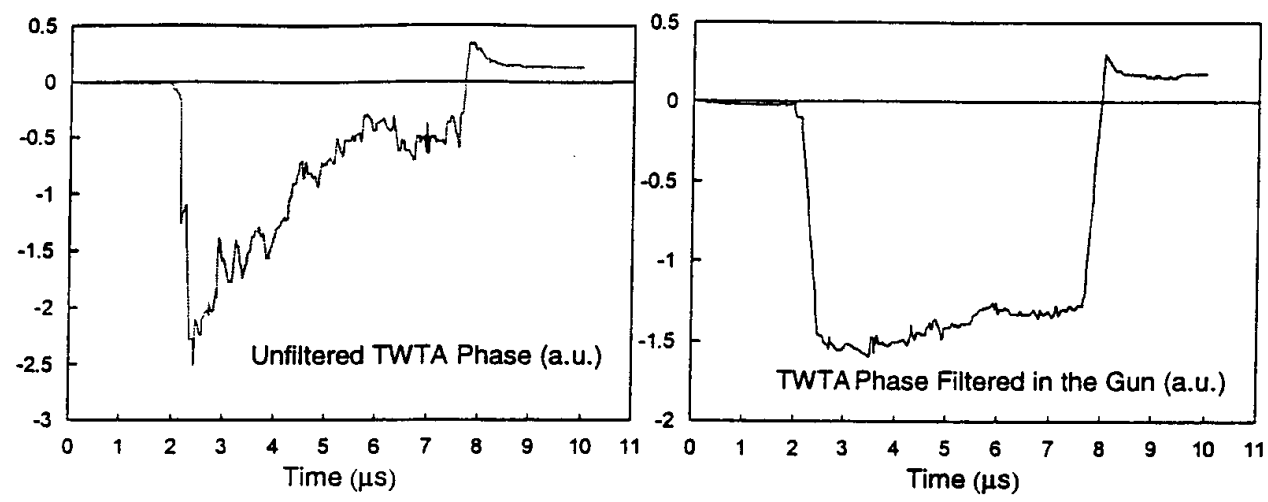

FIGURE 10. Left: unfiltered phase signal at the TWTA output. Right: cleaning up of an individual TWTA RF pulse by high-Q cavity filtering.

A simple initial test of the effect of the accelerator $Q$ on the phase ripple signal was done by feeding the TWTA output signal into the cold test cavity where a field monitor in the full cell was used as the input to a if mixer port. Figure 10 shows that the signal is significantly improved after narrowband filtering by the high $Q$ accelerator cavity.

Based on this preliminary result, we designed an experimental setup, shown in Fig. 11, to produce a filtered $70 \mathrm{MHz}$ signal directly from the high power rf gun. Since the ultimate success of the photoinjector project depends on the proper arrival of the fs laser pulse at the gun cathode, deriving the drive signal for the laser system from the cavity itself can help insure that the laser pulses will adjust to any actual changes in cavity if phase and will eliminate most of the sources of phase noise in the rf system by providing direct synchronization between the laser and if gun fields.

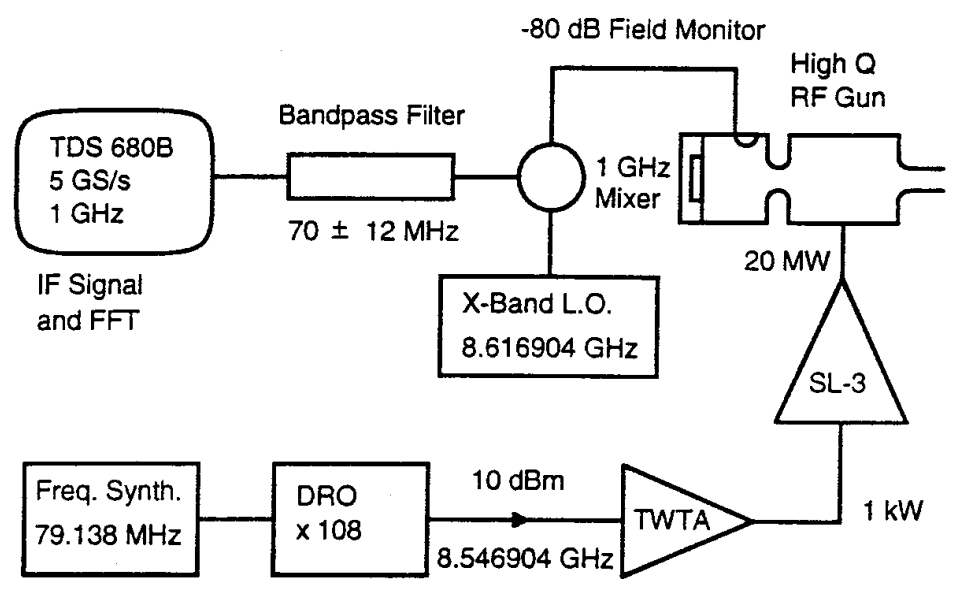

FIGURE 11. Experimental setup for measuring a low noise signal using high-Q cavity filtering. 

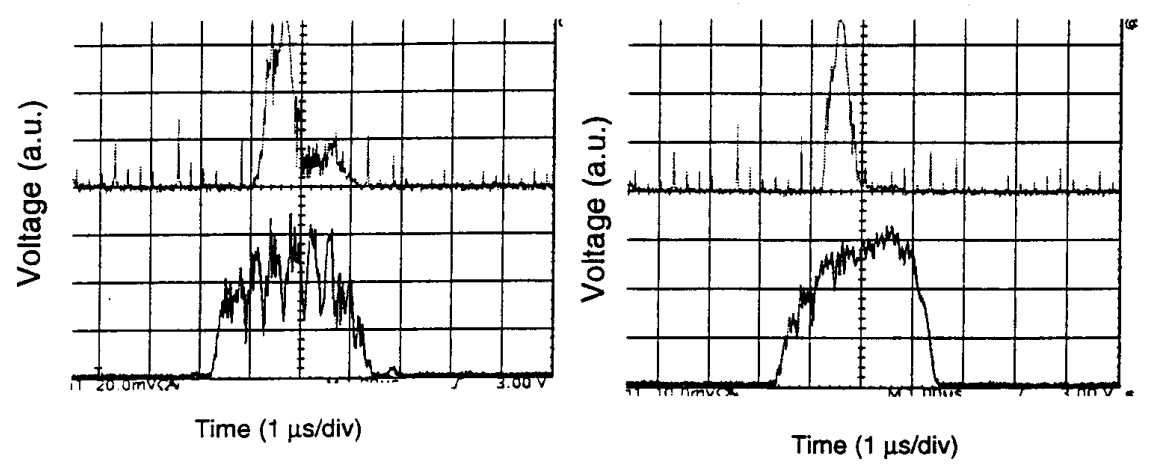

FIGURE 12. Left : the lower trace shows the klystron output pulse before the cavity; the FFT (upper trace) shows noise sidebands. Right : the lower trace shows the high-Q cavity filtering of a klystron output pulse; the corresponding FFT demonstrates noise sideband reduction.

To create the signal, the $-80 \mathrm{~dB}$ field monitor inside the cavity was combined in a 1 $\mathrm{GHz}$ mixer with a local oscillator in the X-band, which differed by $70 \mathrm{MHz}$ from the signal fed into the TWTA. The mixer output then went through a bandpass filter centered at $70 \mathrm{MHz}$ before being displayed along with an FFT spectrum on a $5 \mathrm{GHz}$ bandwidth, $1 \mathrm{Gs} / \mathrm{s}$ digital sampling oscilloscope. $70 \mathrm{MHz}$ was chosen because a bandpass filter at this frequency is high enough to reject noise from the thyratron switch, which ranges in frequency from dc to approximately $20 \mathrm{MHz}$. This technique proved extremely efficient in reducing this and other external noise sources. Figure 12 (left) shows the measurement when the klystron signal was mixed directly with the local oscillator. When the klystron output was used to power the cavity instead, and the field probe signal was mixed with the local oscillator, the $70 \mathrm{MHz}$ signal shown in Fig. 12 (right) was much cleaner. Comparison of the two FFT traces shows that the noise sideband was significantly reduced. In particular, the lowfrequency sideband due to the PFN overmodulation is clearly eliminated.

At this point, where phase noise suppression in the high- $Q$ if gun has been demonstrated, the next question can be addressed: can the filtered signal from the gun be used to drive the semiconductor laser? The main problem here is that we are dealing with a pulsed (1-2 $\mu$ s) signal, and the question of how fast the QW laser modelocks must be resolved. As the semiconductor laser is a high gain system, it is hoped that it can track the phase of a pulsed rf clock quickly. This was first demonstrated by mixing two different local oscillators in a mixer with a $1 \mathrm{GHz}$ frequency difference, but with one of the oscillators modulated by a Hewlett Packard pulser. The resulting clean rf pulses with $<100$ ns rise time was used to drive the $\mathrm{QW}$ laser. Modelocking was achieved with this signal, so the gated oscillator was replaced with the cavity field probe (see Fig. 13). Again, modelocking was achieved at $0.459 \mathrm{GHz}$, as shown in Fig. 14. Thus, direct modelocking of the QW laser system to the rf fields in the energized $\mathrm{X}$-band photoinjector has been demonstrated. 


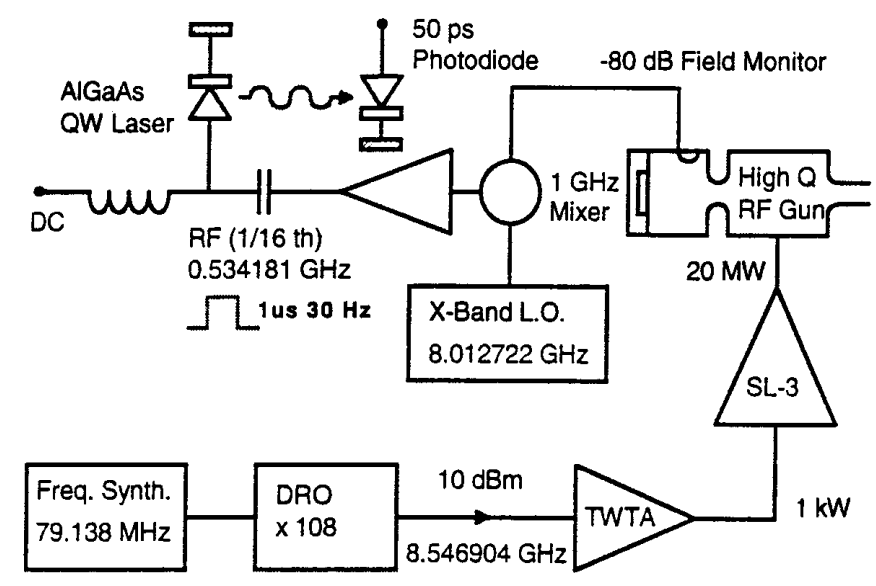

FIGURE 13. Experimental setup for modelocking the semiconductor diode laser off of the cavity fields, at $1 / 16$ th the $\mathrm{X}$-band gun frequency.

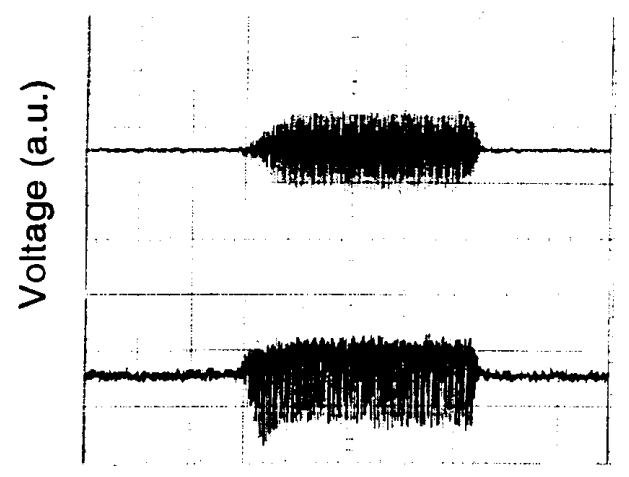

Time (250 ns/div)

FIGURE 14. Modulated rf signal at $459 \mathrm{MHz}$ (upper trace) and photodetector response to the modelocked pulse train (lower trace). 


\section{PHOTOELECTRON PRODUCTION}

A simplified beamline setup was used to produce the first photoelectron signals from the X-band rf gun. UV pulses were sent directly down the beamline to the photocathode, and the resulting photoelectrons were collected by a Faraday cup placed immediately after the gun. The commercial Ti: $\mathrm{Al}_{2} \mathrm{O}_{3}$ system was used to produce pulses at $10 \mathrm{~Hz}$, as described in the previous sections. To simplify synchronization, $\mathrm{rf}$ was derived from the laser oscillator. The phase was coarsely adjusted using a phase delay box. A strong photoelectron signal was seen when within $20^{\circ}$ of an optimum input phase. Figure 15 shows an oscilloscope trace of the $50 \mathrm{~W}$ Faraday cup. The triggering signal, shown on the bottom trace, is the diode signal from a laser back reflection off of the first frequency conversion crystal. Due to timing jitter and amplitude stability of the laser, the large photoelectron spike was not seen at $10 \mathrm{~Hz}$, but it always occurred at the same time with respect to the trigger. The narrowness of the signal is further evidence that the signal is in fact from photoelectrons, as both dark current and plasma discharge electrons produced much longer pulses in the Faraday cup. Finally, when the laser was shuttered from entering the cavity, the photoelectron signal always went away.

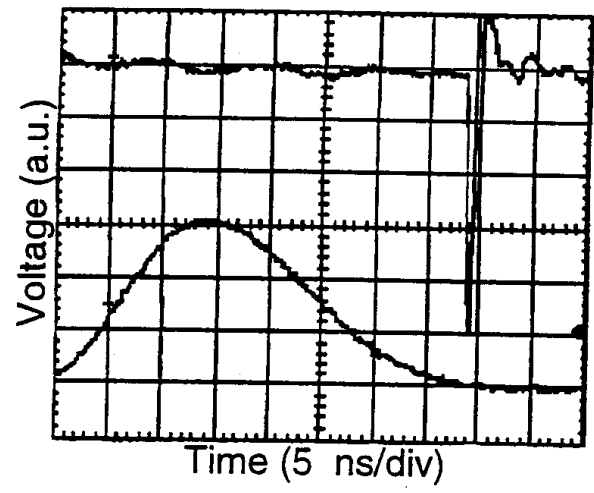

FIGURE 15. Photoelectrons accelerated by the $\mathrm{X}$-band gun appear as a large negative spike in the collected current signal (upper trace). The triggering signal from a slow laser photodiode corresponds to the lower trace.

Future work will improve laser stability so that photoelectrons can be produced reliably at $10 \mathrm{~Hz}$. New diagnostics, including a streak camera, will have to be used on the electron beam, as the photoelectron bunches are too short to be measured in real time by the Faraday cup. In addition, charge, energy, energy spread, emittance, brightness, and stability measurements will be made on the photoinjector electron beam. After the system is fully characterized on a single-shot basis, the AlGaAs diode laser will be installed to create bunches of 100 pulses or more. Once full operation of the photoinjector is achieved, previously proposed FEL and other experiments will take place [1]. 


\section{CONCLUSIONS}

Experimental measurements have been made of the phase noise and jitter characteristics of the X-band photoinjector laser and rf system. The filtering effect of the high $\mathrm{Q}$ structure was demonstrated by comparing klystron and TWT amplifier phase noise with the fields excited in the rf gun. This indicates that the rf gun can be used as a master oscillator, and could be energized by either a RF oscillator such as a magnetron or a compact source such as a cross-field amplifier. The X-band gun if fields were then used to drive a synchronously modelocked AlGaAs QW laser, thus providing the first experimental demonstration of direct synchronization between a short pulse laser system and the rf fields excited in a high $Q$ if photoinjector. This novel, $\mathrm{GHz}$ repetition rate laser system is being developed to replace the more conventional femtosecond Ti: $\mathrm{Al}_{2} \mathrm{O}_{3}$ system. Finally, dark current measurements and initial photoelectron measurements have been made. This represents the first operation of a high gradient $\mathrm{X}$-band photoinjector.

Future experiments will include a detailed study of the chirped pulse FEL interaction [1], where ultrawideband pulses of millimeter-wave radiation will be produced at MW power levels, as well as ultrahigh intensity Compton and Kapitza-Dirac scattering and relativistic ponderomotive scattering.

\section{ACKNOWLEDGMENTS}

This work is supported in part by DoD/AFOSR (MURI) F49620-95-1-0253, AFOSR (ATRI) F30602-94-2-001, and ARO DAAHO4-95-1-033. This work was also partially supported under the auspices of the U.S. Department of Energy by the Lawrence Livermore National Laboratory under Contract No. W-7405-ENG-48 through the Institute for Laser Science and Applications. We would like to thank G. Cruz, F.B. Gensheer, C.V. Bennett, G.P. LeSage, and T. Hillyer for their assistance.

\section{REFERENCES}

1. G. P. Le Sage et al., "Theory and design of a photoinjector-driven chirped pulse free-electron maser," IEEE Transactions on Plasma Science, vol. 24, pp. 781-795, 1996.

2. C. Travier, "RF guns: Bright injectors for FEL," Nucl. Instrum. Methods Phys. Res., vol. A304, p. $285,1991$.

3. S. C. Chen et al., "High gradient acceleration in a $17 \mathrm{GHz}$ photocathode RF gun," in Proc. 1993 PAC, vol. 4, p. 2546, 1993.

4. E. Esarey, P. Sprangle, J. Krall, and A. Ting, " Overview of plasma-based accelerator concepts," IEEE Transactions on Plasma Science, vol. 24, pp. 252-288, 1996.

5. F. V. Hartemann and A. K. Kerman, "Classical theory of nonlinear Compton scattering," Phys Rev. Lett. 76, 627 (1996).

6. F. V. Hartemann et al., "Spectral analysis of the nonlinear relativistic Doppler shift in ultrahigh intensity Compton scattering," Phys Rev. E 54, 2956 (1996). 
7. C. Bula et al., "Observation of nonlinear effects in Compton scattering," Phys Rev. Lett. 76, 3116 (1996).

8. A. Gover et al., "Time and frequency domain analysis of superradiant coherent synchotron radiation in a waveguide free-electron laser," Phys. Rev. Lett., vol. 72, p. 1192, 1994.

9. F. V. Hartemann et al., "Coherent synchotron radiation in a cylindrical waveguide with a helical wiggler," Phys. Plasmas, vol. 1, p. 1306, 1994.

10. F. V. Hartemann et al., "Transform-limited coherent synchotron wavepackets in a chirped pulser free-electron laser," Physics of Plasmas 3, 2446, 1996.

11. N. C. Currie and C. E. Brown, Eds. Principles and Applications of Millimeter-Wave Radar. Norwood, MA: Artech House, 1987.

12. National Research Council, Free Electron Lasers and Other Advanced Sources of Light. Washington, D.C.: National Academy, 1994.

13. F. V. Hartemann, et. al., "Nonlinear ponderomotive scattering of relativistic electrons by an intense laser field at focus," Phys Rev. E 51, 4833, 1995.

14. G. Malka, E. Levebvre, and J. L. Miquel, "Experimental observation of electrons accelerated in vacuum to relativistic energies by a high-intensity laser," Phys. Rev. Lett. 78, 3314, 1997.

15. P. L. Kapitza and P. A. M. Dirac, "The reflection of electrons from standing light waves," Proc. Cambridge Philos. Soc. 29, 297, 1933.

16. P. H. Bucksbaum, L. D. Van Woerkom, R. R. Freemann, and D. W. Schumacher, "Nonresonant above-threshold ionization by circularly polarized subpicosecond pulses," Phys. Rev. A 41, 4119, 1990.

17. H. D. Hagstrum, "Surface Physics," in Physics Vade Mecum, H. L. Anderson, Ed. New York: Amer. Inst. Phys., 1989, pp. 418-335.

18. P. J. Delfyett et al., "High power ultrafast laser diodes," IEEE J. Quantum Electron., vol. 28, p. 2203, 1992.

19. A. J. Taylor et al., "Timing jitter in model-locked and gain-switched InGaAsP injection lasers," Appl. Phys. Let., vol. 48, p. 681, 1986.

20. D. J. Derickson, P. A. Morton, and J. E. Bowers, "Comparison of timing jitter in external and monolithic cavity mode-locked semiconductor lasers," IEEE J. Quantum Electron., vol. 28, p. 2186, 1992. 